\title{
Inferior colliculus lesions impair eyeblink conditioning in rats
}

\author{
John H. Freeman, ${ }^{1}$ Hunter E. Halverson, and Erin M. Hubbard \\ Department of Psychology, University of lowa, lowa City, lowa 52242, USA
}

\begin{abstract}
The neural plasticity necessary for acquisition and retention of eyeblink conditioning has been localized to the cerebellum. However, the sources of sensory input to the cerebellum that are necessary for establishing learning-related plasticity have not been identified completely. The inferior colliculus may be a source of sensory input to the cerebellum through its projection to the medial auditory thalamus. The medial auditory thalamus is necessary for eyeblink conditioning in rats and projects to the lateral pontine nuclei, which then project to the cerebellar nuclei and cortex. The current experiment examined the role of the inferior colliculus in auditory eyeblink conditioning. Rats were given bilateral or unilateral (contralateral to the conditioned eye) lesions of the inferior colliculus prior to $10 \mathrm{~d}$ of delay eyeblink conditioning with a tone CS. Rats with bilateral or unilateral lesions showed equivalently impaired acquisition. The extent of damage to the contralateral inferior colliculus correlated with several measures of conditioning. The findings indicate that the contralateral inferior colliculus provides auditory input to the cerebellum that is necessary for eyeblink conditioning.
\end{abstract}

Pavlovian eyeblink conditioning has been used extensively as a model system for examining the neurobiological mechanisms of learning and memory (Thompson 2005). Eyeblink (eyelid and nictitating membrane movement) conditioning typically consists of presentations of a tone or light conditioned stimulus (CS) that is paired with an unconditioned stimulus (US) that elicits the blink reflex before training (Gormezano et al. 1962; Schneiderman et al. 1962). In delay conditioning, the onset of the US follows the onset of the CS, and both stimuli terminate at the same time. The neural plasticity that is necessary for acquisition and retention of delay eyeblink conditioning has been localized to the cerebellum (Thompson 2005; Ohyama et al. 2006). However, the neural pathways that provide sensory input to the cerebellum during eyeblink conditioning have not been fully delineated. Identification of the sensory input pathways that are necessary for cerebellar learning is a critical step toward developing a comprehensive understanding of cerebellar function.

A strong case has been made that the pontine mossy fiber projection to the cerebellum is the proximal end of the sensory input pathway for a CS during eyeblink conditioning. Lesions of the middle cerebellar peduncle impair acquisition and retention of eyeblink conditioning (Lewis et al. 1987). Lesions or inactivation of the lateral pontine nuclei also impair retention (Steinmetz et al. 1987; Knowlton and Thompson 1988). Neurophysiological studies have shown that lateral pontine neurons are activated by auditory input and pontine stimulation elicits short-latency evoked responses in the cerebellum (Steinmetz et al. 1987; Gould et al. 1993; Freeman and Muckler 2003). Moreover, stimulation of the mossy fiber pathway as a CS has been shown to be sufficient for eyeblink conditioning (Steinmetz et al. 1986, 1987, 1989; Steinmetz 1990; Tracy et al. 1998; Hesslow et al. 1999; Bao et al. 2000; Freeman and Rabinak 2004; Freeman et al. 2005). The neuroanatomical and neurophysiological studies of the role of pontine mossy fibers in eyeblink conditioning clearly indicate that they are the proximal end of the CS pathway.

An unresolved issue is the origin of sensory input to the pontine nuclei that is necessary for eyeblink conditioning. A component of the auditory input to the pontine nuclei comes

\footnotetext{
1 Corresponding author.
}

E-mail john-freeman@uiowa.edu; fax (319) 335-0191.

Article is online at http://www.learnmem.org/cgi/doi/10.1101//m.716107. from a monosynaptic projection from the cochlear nuclei (Steinmetz et al. 1987; Gould et al. 1993; Campolattaro et al. 2007). The monosynaptic pathway from the cochlear nuclei provides auditory CS information to the pontine nuclei, but it might not be sufficient for acquisition of eyeblink conditioning. Neurons in the pons that receive input from the cochlear nuclei show response latencies of 3-5 msec after the onset of an auditory stimulus (Steinmetz et al. 1987; Gould et al. 1993). However, an interstimulus interval (ISI) $>50 \mathrm{msec}$ is required for acquisition of eyeblink conditioning (Smith et al. 1969). Other auditory structures including the inferior colliculus and thalamus may be critically involved in relaying tone CS information to the pontine nuclei after $5 \mathrm{msec}$.

The medial auditory thalamic nuclei (MATN; medial division of the medial geniculate, posterior intralaminar nucleus, and suprageniculate) have been shown to play a critical role in acquisition of eyeblink conditioning in rats (Halverson and Freeman 2006; Campolattaro et al. 2007). Unilateral lesions of the MATN contralateral to the conditioned eye severely impaired acquisition of eyeblink conditioning using a tone CS but had no effect on acquisition of conditioning using a light CS in rats (Halverson and Freeman 2006). In another study, stimulation of the MATN was a highly effective CS when paired with a peripheral US (Campolattaro et al. 2007). This study also found that the MATN projects directly to the lateral pontine nuclei. Single unit activity in the medial geniculate recorded during differential auditory trace conditioning showed CS-related and CR-related facilitation in the dorsal and medial nuclei, with the most robust facilitation occurring in the medial nucleus ( $\mathrm{O}^{\prime}$ Connor et al. 1997). The medial auditory thalamus may, therefore, be one of the components of the CS pathway for auditory cerebellar learning.

Auditory input to the MATN comes primarily from the inferior colliculus (IC) (LeDoux et al. 1987; Campolattaro et al. 2007). The MATN also project back to the IC, but this auditory feedback is not necessary for eyeblink conditioning (Campolattaro et al. 2007). Moreover, the direct projection from the IC to the pontine nuclei is also not necessary for eyeblink conditioning (Campolattaro et al. 2007). The IC may, therefore, play a role in eyeblink conditioning through its projection to the MATN. Evidence supporting a possible role for the IC in eyeblink condition- 
ing comes from experiments that used IC stimulation as a CS for nictitating membrane conditioning in rabbits (Patterson 1969, 1970; Nowak et al. 1999). However, the effects of lesions or inactivation of the IC on eyeblink conditioning using a tone CS have not been published. The current experiment examined the effects of bilateral or unilateral lesions of the inferior colliculus on auditory eyeblink conditioning in rats.

\section{Results}

Both lesion groups sustained damage throughout the rostralcaudal extent of the IC. The extent of damage ranged from $60.5 \%$ to $93.8 \%$ in the group given bilateral lesions and $36.5 \%$ to $87.1 \%$ in the group given unilateral lesions. In both groups, the spared IC was in the more medial and ventral areas of the IC. Figure 1 displays drawings of bilateral and unilateral lesions. The largest lesions were very substantial, but sparing of the most ventral IC was observed in every rat. Some of the rats with large IC lesions also sustained damage in the periaqueductal gray (Fig. 1).

Bilateral or unilateral lesions of the IC resulted in impaired acquisition of eyeblink conditioning (Fig. 2). The impairment was evident in the CR percentage and CR amplitude measures. No differences were observed between the groups that were given bilateral or unilateral lesions. The absence of a difference between the lesion groups suggested that the ipsilateral IC, damaged only in the group given bilateral lesions, does not play a role in the acquisition of auditory eyeblink conditioning.

ANOVAs of the CR percentage and CR amplitude data yielded significant main effects for the group factor, $F_{(2,40)}=3.65$, $P<0.05$ for percentage and $F_{(2,40)}=3.86, P<0.05$ for amplitude. The group-by-session interactions were not significant. The main effects were due to better performance in the control group relative to both lesion groups, which did not differ $(P<0.05)$.

The relationship between the extent of the lesions and eyeblink conditioning was assessed in a series of analyses. Because the lesion groups were equally impaired, the analyses of lesion extent used data from only the IC contralateral to the conditioned eye. When the combined lesion group was subdivided based on the amount of damage to the contralateral IC, it was evident that only rats with nearly complete lesions were impaired (Fig. 3). Figure 3 displays the data from the lesion groups
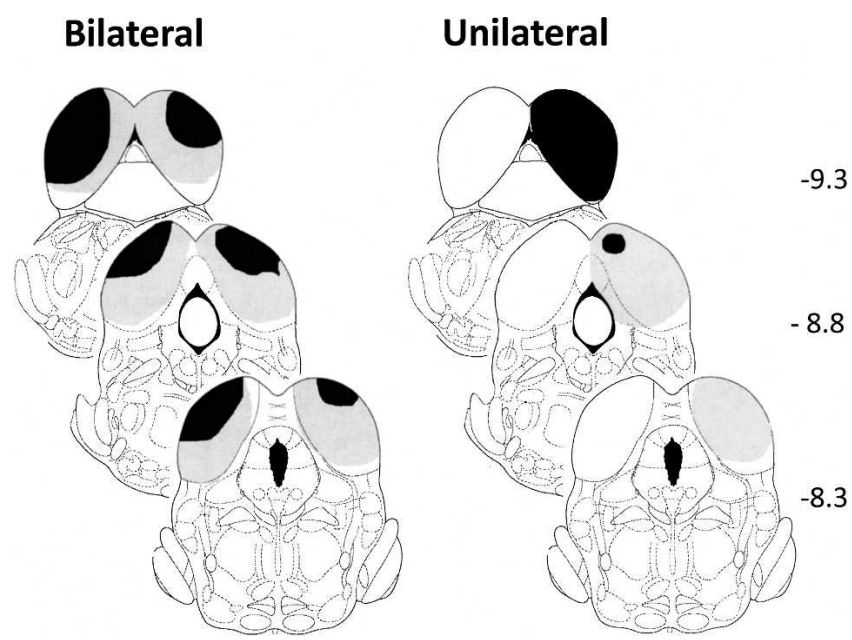

Figure 1. Inferior colliculus lesions. The largest (gray shaded areas) and smallest (black shaded areas) bilateral (left) and unilateral (right) lesions of the inferior colliculus (IC). The unilateral lesions were contralateral to the conditioned eye. Numbers indicate the anterior-posterior stereotaxic coordinates of the sections relative to bregma (Paxinos and Watson 1998).

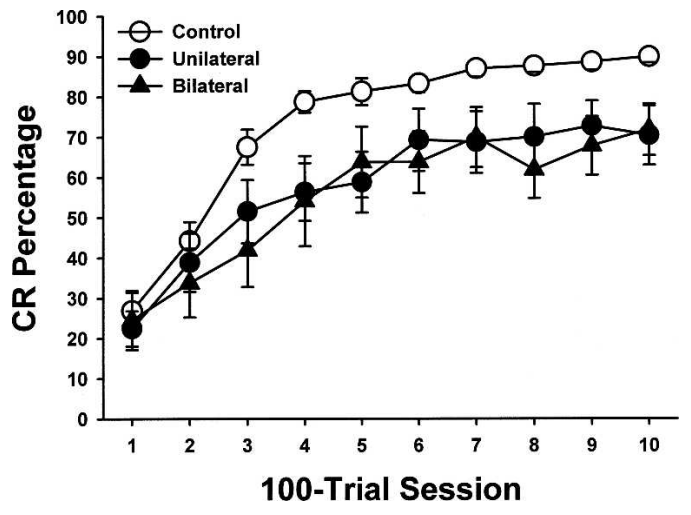

Figure 2. Bilateral and unilateral lesions of the inferior colliculus impair eyeblink conditioning. Mean $( \pm$ ) SEM conditioned response $(C R)$ percentage for rats given control surgery (white circles), unilateral inferior colliculus lesions (black circles), and bilateral inferior colliculus lesions (black triangles) across 10100 -trial sessions of eyeblink conditioning.

separated into subgroups with large $(0 \%-20 \%$ of control, $n=7)$, medium $(21 \%-40 \%$ of control, $n=15)$, or small $(41 \%-63 \%$ of control, $n=6$ ) lesions of the contralateral IC. The group with large lesions was severely impaired, whereas the groups with medium or small lesions were virtually unimpaired.

To further assess the relationship between lesion extent and eyeblink conditioning, several regression analyses were performed. The dependent variables for the regression analyses included the mean percentage of CRs across all 10 training sessions, the percentage of CRs at the end of training, the number of sessions to reach a performance criterion of $70 \%$ CRs (failure to reach $70 \%$ resulted in a score of 10 ), and the difference between the percentage of CRs during the first and last training sessions. The results of the regression analyses are displayed in Table 1 using the ANOVA format. Significant relationships were found for the extent of remaining IC and the overall percentage of CRs (Fig. 4A), the percentage of CRs during the last training session, and the number of sessions to reach criterion (Fig. 4B). The regression analysis for the change in CR percentage from sessions 1 to 10 was not significant.

\section{Discussion}

Bilateral and unilateral (contralateral to the conditioned eye) lesions of the inferior colliculus impaired acquisition of eyeblink conditioning to a similar degree (Fig. 2). The dorsal and caudal IC was damaged severely in both lesion groups, with sparing of the rostral-ventral IC (Fig. 1). Rats with the largest lesions (81\%$93.8 \%$ ) were severely impaired, while rats with smaller lesions were unimpaired (Fig. 3). There was a linear relationship between the extent of contralateral IC damage and measures of conditioning including the overall percentage of CRs, the percentage of CRs during the final training session (session 10), and the number of sessions to reach a performance criterion of $70 \%$ CRs in a training session (Table 1).

Unilateral IC lesions produced a deficit that was nearly identical to the deficit observed in rats with bilateral IC lesions. The unilateral IC lesions were made contralateral to the conditioned eye. The contralateral IC projects ipsilaterally to the medial auditory thalamus, which then projects ipsilaterally to the lateral pontine nuclei (contralateral to the conditioned eye) (Campolattaro et al. 2007). Lesions of the MATN contralateral to the conditioned eye completely blocked acquisition of auditory eyeblink conditioning, probably by removing its input to the pontine nuclei (Halverson and Freeman 2006). Moreover, stimulation of the 


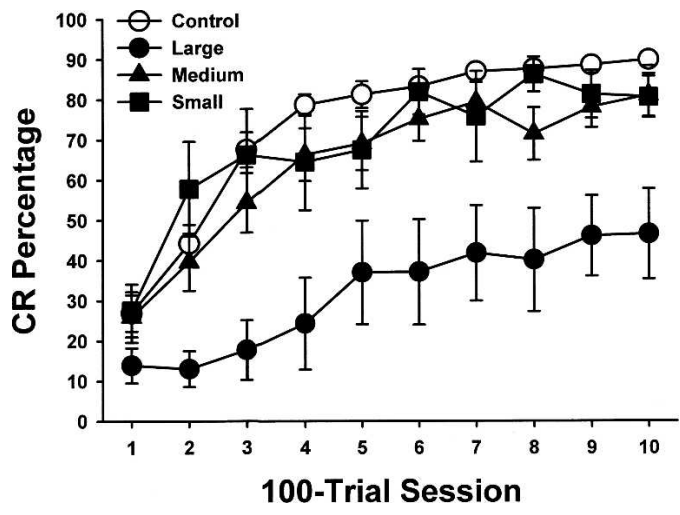

Figure 3. Eyeblink conditioning as a function of the relative size of inferior colliculus (IC) lesions. Mean ( \pm ) conditioned response (CR) percentage for rats given control surgery (white circles), large IC lesions (black circles), medium IC lesions (black triangles), and small IC lesions (black squares) across 10 100-trial sessions of eyeblink conditioning.

contralateral MATN was a highly effective CS when paired with a peripheral US (Campolattaro et al. 2007). The findings of the current study and previous studies (Halverson and Freeman 2006; Campolattaro et al. 2007) indicate that the auditory CS pathway in eyeblink conditioning is unilateral from the IC to the cerebellum. We have no information regarding the CS pathway within the auditory nuclei that are afferent to the IC, but it is likely that bilateral input from the cochlear nuclei, nucleus of the lateral lemniscus, and the superior olive plays a role in auditory eyeblink conditioning.

The medial and ventral areas of the IC that were not destroyed in the rats given lesions and other auditory areas appear to be sufficient for eyeblink conditioning in most of the rats in the current experiment. The ventral part of the central IC in intact rats contains neurons that respond to sound frequencies well above the 2- and 8-kHz tones used in this experiment (Ryan et al. 1988). However, the neuronal responses in the spared IC could have been modified by training and might have also been reorganized to compensate for the damaged areas. As a result, the spared neurons in the central IC might have been able to support conditioning with 2 - or $8-\mathrm{kHz}$ tones, even though the neurons in this area would normally be less sensitive to these relatively low sound frequencies. Neurons in the external IC, which have broad tuning properties (Aitkin et al. 1975), were spared in many cases and may have been able to support conditioning. It is also possible that conditioning in the lesioned rats was supported by projections from the cochlear nuclei directly to the medial auditory thalamus (Malmierca et al. 2002) or the direct projection from the ventral cochlear nucleus to the pontine nuclei (Steinmetz et al. 1987; Campolattaro et al. 2007). The most reasonable hypothesis at this point is that the combination of spared IC neurons and cochlear nucleus projections supported eyeblink conditioning by providing input to the medial auditory thalamus and pontine nuclei in the rats with IC lesions.

Table 1. Regression analysis and Pearson's $r$ for IC size versus the behavioral measures

\begin{tabular}{lrcr}
\hline Variables & F-ratio & P-value & Pearson's r \\
\hline CR \% & 10.40 & 0.003 & 0.535 \\
CR \% last session & 5.29 & 0.03 & 0.411 \\
Difference: CR \% s1 versus S10 & 2.49 & 0.127 & 0.295 \\
Sessions to criterion & 11.00 & 0.003 & -0.545 \\
\hline
\end{tabular}

The exact role of the IC in eyeblink conditioning has not been determined. Neuronal activity within the IC during nictitating membrane conditioning in rabbits showed exclusively sensory responses to the tone CS and the sound of the airpuff US (McCormick et al. 1983). Neuronal responses to an auditory CS have also been examined during nictitating membrane conditioning using a white noise CS that was presented at the threshold for eliciting CRs in well-trained rabbits (Kettner and Thompson 1982). No differences were found in neuronal activity within the IC between training trials with and without CRs, suggesting that the IC was not involved in signal detection or priming the behavioral response. The findings of the McCormick et al. (1983) and Kettner and Thompson (1982) studies indicated that the IC serves primarily as a sensory relay during nictitating membrane conditioning rather than as a site of plasticity (Kettner and Thompson 1985). It is likely that the IC plays the same role in rodent eyeblink conditioning, but additional neurophysiological studies are necessary to validate this claim.

The results of this experiment indicate that the IC plays an important role in auditory eyeblink conditioning. Our previous study found direct projections from widespread regions of the IC to the medial auditory thalamic nuclei (Campolattaro et al. 2007). It is likely, therefore, that the IC provides a substantial proportion of the auditory input to the medial auditory thalamus that is necessary for eyeblink conditioning. Other auditory inputs to the thalamus such as the direct projection from the cochlear nuclei may provide additional parallel input during auditory eyeblink conditioning.
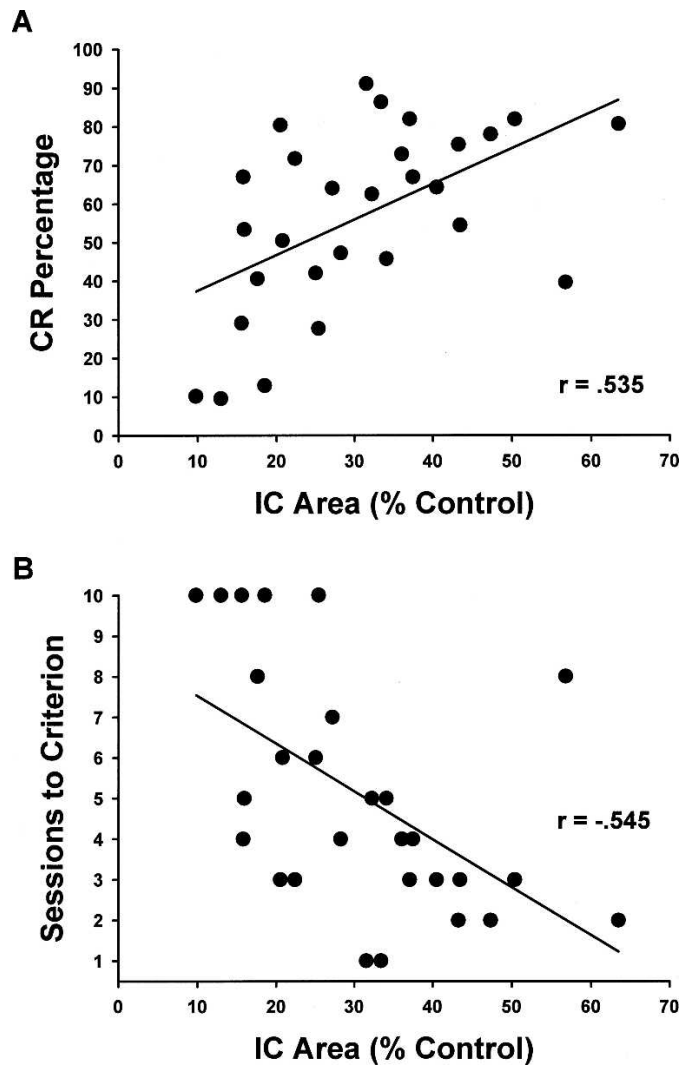

Figure 4. Scatterplots with regression lines showing the relationship between lesion size and two measures of conditioning. Area of the IC in the rats given lesions is plotted against $(A)$ the overall conditioned response (CR) percentage and sessions to reach $(B)$ a performance criterion of $70 \% \mathrm{CR}$. 


\section{Materials and Methods}

\section{Subjects}

Subjects were 43 adult Long Evans male rats (250-350 g), 150 d of age at the beginning of the experiment. The rats were housed in Spence Laboratories of the Psychology at the University of Iowa with a 12-h light-dark cycle, with light onset at 7:00 a.m.

\section{Groups}

Rats were assigned to groups that received bilateral lesions of the inferior colliculus $(n=11)$, unilateral lesions of the inferior colliculus that were contralateral to the conditioned eye $(n=17)$, or a control surgery $(n=15)$.

\section{Surgery}

One week prior to training, rats were removed from their home cage and anesthetized by an i.p. injection of sodium pentobarbital $(80 \mathrm{mg} / \mathrm{kg})$. An i.p. injection of atropine sulfate $(0.45 \mathrm{mg} / \mathrm{kg})$ was administered to reduce respiratory tract secretions. After the onset of anesthesia, lesions of the inferior colliculus were produced by passing $1.0 \mathrm{~mA}$ of DC current for $10 \mathrm{sec}$ in each of three sites per side through an insect pin insulated with epoxylite. The stereotaxic coordinates were $8.3,8.8$, and $9.2 \mathrm{~mm}$ posterior to bregma; $2.2,2.0$, and $1.9 \mathrm{~mm}$ lateral to midline; and 4.2, 4.6, and $4.3 \mathrm{~mm}$ below the skull surface. Skull holes were sealed with bone wax. Rats in the control group had electrodes lowered into the inferior colliculus either bilaterally $(n=6)$ or unilaterally $(n=9)$, but no current was passed.

All of the rats were implanted with differential electromyographic (EMG) electrodes in the left upper eyelid muscle (orbicularis oculi) and a ground electrode attached to a stainless steel skull screw. The EMG electrode leads terminated in gold pins held in a plastic connector, which was secured to the skull with dental acrylic. A bipolar stimulating electrode (Plastics One, Inc.) for delivering the shock US was implanted immediately caudal to the left eye. The bipolar electrode terminated in a plastic connector that was secured to the skull with dental acrylic.

\section{Conditioning apparatus}

The conditioning apparatus consisted of a small-animal sound attenuation chamber (BRS/LVE) with a small-animal operant chamber (BRS/LVE) contained inside. The rats were kept in the operant chamber during conditioning. Cables with connectors for the EMG and US electrodes were attached to a commutator. The electrode leads from the rat's head stage were connected to peripheral equipment and a desktop computer. Computer software controlled the delivery of stimuli and the recording of eyelid EMG activity (JSA Designs). The US was delivered through a stimulus isolator (World Precision Instruments). EMG activity was recorded differentially, filtered $(500-5000 \mathrm{~Hz})$, amplified $(2000 \times)$, and integrated (time constant $=20 \mathrm{msec}$ ).

\section{Eyeblink conditioning}

Rats were given 10 paired training sessions. The paired training sessions consisted of 100 trials, each with 90 trials of a tone CS (500 msec, 2 or $8 \mathrm{kHz}, 88 \mathrm{~dB}$ SPL) paired with a shock US (25 msec, $2.5-4.0 \mathrm{~mA}$ ) and $10 \mathrm{CS}$-alone trials, occurring on every tenth trial. The CS-alone trials were included in order to assess behavioral responses (integrated EMG activity) uncontaminated by URs. The interstimulus interval for paired trials was $475 \mathrm{msec}$. Trials were separated by an intertrial interval that averaged 30 sec. Behavioral data were examined from computer records of EMG responses. CRs were defined as responses that crossed a threshold of $0.4 \mathrm{~V}$ above the baseline activity during the CS period, but at least $80 \mathrm{msec}$ after CS onset, to avoid contamination of the CR measures by the startle (alpha) response. The amplitude, onset latency, and peak latency of the CR were measured on CS-alone trials in which a CR occurred.

\section{Histology}

After training was completed the rats were euthanized with a lethal injection of sodium pentobarbital $(90 \mathrm{mg} / \mathrm{kg})$ and transcardially perfused with $0.1 \mathrm{M}$ PBS, followed by $10 \%$ formalin. The brains were post-fixed in formalin for $2 \mathrm{~d}$ and then placed in a solution of $30 \%$ sucrose in formalin before sectioning. The brains were sectioned in the coronal plane at $50 \mu \mathrm{m}$ with a sliding microtome. Sections were then stained with thionin. The extent of the inferior colliculus lesions was determined by measuring the area of remaining tissue in three sections corresponding to plates 53, 55, and 57 in Paxinos and Watson's stereotaxic atlas (Paxinos and Watson 1998) in each rat. Area was measured using NIH image after manually outlining the remaining inferior colliculus in each section. The amount of remaining tissue in the inferior colliculus was then converted to a percentage of the composite area of the control group.

\section{Acknowledgments}

This work was supported by grants from the NIMH (MH065483, MH080005) and NINDS (NS038890).

\section{References}

Aitkin, L.M., Webster, W.R., Veale, J.L., and Crosby, D.C. 1975. Inferior colliculus. I. Comparison of response properties of neurons in central, pericentral, and external nuclei of adult cat. J. Neurophysiol. 38: 1196-1207.

Bao, S., Chen, L., and Thompson, R.F. 2000. Learning- and cerebellum-dependent neuronal activity in the lateral pontine nucleus. Behav. Neurosci. 114: 254-261.

Campolattaro, M.M., Halverson, H.E., and Freeman, J.H. 2007. Medial auditory thalamic stimulation as a conditioned stimulus for eyeblink conditioning in rats. Learn. Mem. 14: 152-159.

Freeman Jr., J.H. and Muckler, A.S. 2003. Developmental changes in eyeblink conditioning and neuronal activity in the pontine nuclei. Learn. Mem. 10: 337-345.

Freeman, J.H. and Rabinak, C.A. 2004. Eyeblink conditioning in rats using pontine stimulation as a conditioned stimulus. Integr. Physiol. Behav. Sci. 39: 180-191.

Freeman, J.H., Rabinak, C.A., and Campolattaro, M.M. 2005. Pontine stimulation overcomes developmental limitations in the neural mechanisms of eyeblink conditioning. Learn. Mem. 12: 255-259.

Gormezano, I., Schneiderman, N., Deaux, E.G., and Fuentes, I. 1962. Nictitating membrane: Classical conditioning and extinction in the albino rabbit. Science 138: 33-34.

Gould, T.J., Sears, L.L., and Steinmetz, J.E. 1993. Possible CS and US pathways for rabbit classical eyelid conditioning: Electrophysiological evidence for projections from the pontine nuclei and inferior olive to cerebellar cortex and nuclei. Behav. Neural Biol. 60: 172-185.

Halverson, H.E. and Freeman, J.H. 2006. Medial auditory thalamic nuclei are necessary for eyeblink conditioning. Behav. Neurosci. 120: $880-887$.

Hesslow, G., Svensson, P., and Ivarsson, M. 1999. Learned movements elicited by direct stimulation of cerebellar mossy fiber afferents. Neuron 24: 179-185.

Kettner, R.E. and Thompson, R.F. 1982. Auditory signal detection and decision processes in the nervous system. J. Comp. Physiol. Psychol. 96: 328-331.

Kettner, R.E. and Thompson, R.F. 1985. Cochlear nucleus, inferior colliculus, and medial geniculate responses during the behavioral detection of threshold-level auditory stimuli in the rabbit. J. Acoust. Soc. Am. 77: 2111-2127.

Knowlton, B.J. and Thompson, R.F. 1988. Microinjections of local anesthetic into the pontine nuclei reduce the amplitude of the classically conditioned eyelid response. Physiol. Behav. 43: 855-857.

LeDoux, J.E., Ruggiero, D.A., Forest, R., Stornetta, R., and Reis, D.J. 1987. Topographical organization of convergent projections to the thalamus from the inferior colliculus and spinal cord of the rat. $J$. Comp. Neurol. 264: 123-146.

Lewis, J.L., LoTurco, J.J., and Solomon, P.R. 1987. Lesions of the middle cerebellar peduncle disrupt acquisition and retention of the rabbit's classically conditioned nictitating membrane response. Behav. Neurosci. 101: 151-157.

Malmierca, M.S., Merchan, M.A., Henkel, C.K., and Oliver, D.L. 2002. Direct projections from cochlear nuclear complex to auditory thalamus in the rat. J. Neurosci. 22: 10891-10897.

McCormick, D.A., Lavond, D.G., and Thompson, R.F. 1983. Neuronal 
responses of the rabbit brainstem during performance of the classically conditioned nictitating membrane (NM)/eyelid response. Brain Res. 271: 73-88.

Nowak, A.J., Kehoe, E.J., Macrae, M., and Gormezano, I. 1999. Conditioning and reflex modification of the rabbit nictitating membrane response using electrical stimulation in auditory nuclei. Behav. Brain Res. 105: 189-198.

O'Connor, K.N., Allison, T.L., Rosenfield, M.E., and Moore, J.W. 1997. Neural activity in the medial geniculate nucleus during auditory trace conditioning. Exp. Brain Res. 113: 534-556.

Ohyama, T., Nores, W.L., Medina, J.F., Riusech, F.A., and Mauk, M.D. 2006. Learning-induced plasticity in deep cerebellar nucleus. $J$. Neurosci. 26: 12656-12663.

Patterson, M.M. 1969. The effects of intracranial CS and fluctuating ISI on the classically conditioned rabbit nictitating membrane response. Ph.D. thesis, University of Iowa, Iowa City, IA.

Patterson, M.M. 1970. Classical conditioning of the rabbit's (oryctolagus cuniculus) nictitating membrane response with fluctuating ISI and intracranial CS. J. Comp. Physiol. Psychol. 72: 193-202.

Paxinos, G. and Watson, C. 1998. The rat brain in stereotaxic coordinates. Academic Press, New York.

Ryan, A.F., Furlow, Z., Woolf, N.K., and Keithley, E.M. 1988. The spatial representation of frequency in the rat dorsal cochlear nucleus and inferior colliculus. Hear. Res. 36: 181-190.

Schneiderman, N., Fuentes, I., and Gormezano, I. 1962. Acquisition and extinction of the classically conditioning eyelid response in the albino rabbit. Science 136: 650-652.

Smith, M.C., Coleman, S.R., and Gormezano, I. 1969. Classical conditioning of the rabbit's rictitating membrane response at backward, simultaneous, and forward CS-US intervals. J. Comp. Physiol. Psychol. 2: 226-231.

Steinmetz, J.E. 1990. Neuronal activity in the rabbit interpositus nucleus during classical NM-conditioning with a pontine-nucleus-stimulation CS. Psychol. Sci. 1: 378-382.

Steinmetz, J.E., Rosen, D.J., Chapman, P.F., Lavond, D.G., and

Thompson, R.F. 1986. Classical conditioning of the rabbit eyelid response with a mossy fiber stimulation CS. I. Pontine nuclei and middle cerebellar peduncle stimulation. Behav. Neurosci. 100: $878-887$.

Steinmetz, J.E., Logan, C.G., Rosen, D.J., Thompson, J.K., Lavond, D.G., and Thompson, R.F. 1987. Initial localization of the acoustic conditioned stimulus projection system to the cerebellum essential for classical eyelid conditioning. Proc. Natl. Acad. Sci. 84: 3531-3535.

Steinmetz, J.E., Lavond, D.G., and Thompson, R.F. 1989. Classical conditioning in rabbits using pontine nucleus stimulation as a conditioned stimulus and inferior olive stimulation as an unconditioned stimulus. Synapse 3: 225-233.

Thompson, R.F. 2005. In search of memory traces. Annu. Rev. Psychol. 56: $1-23$.

Tracy, J.A., Thompson, J.K., Krupa, D.J., and Thompson, R.F. 1998. Evidence of plasticity in the pontocerebellar conditioned stimulus pathway during classical conditioning of the eyeblink response in the rabbit. Behav. Neurosci. 112: 267-285.

Received July 24, 2007; accepted in revised form October 12, 2007. 


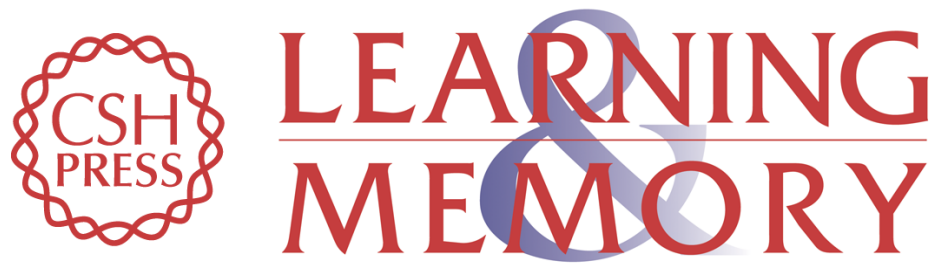

\section{Inferior colliculus lesions impair eyeblink conditioning in rats}

John H. Freeman, Hunter E. Halverson and Erin M. Hubbard

Learn. Mem. 2007, 14:

Access the most recent version at doi:10.1101//m.716107

References This article cites 30 articles, 8 of which can be accessed free at: http://learnmem.cshlp.org/content/14/12/842.full.html\#ref-list-1

License

Email Alerting Receive free email alerts when new articles cite this article - sign up in the box at the Service top right corner of the article or click here. 